\title{
Human herpesvirus type 8 in tuberculosis patients with effusion
}

\author{
Shih-Ming Tsao ${ }^{1,2}$, Chun-Liang Lai ${ }^{3,4}$, Ming-Nan Lin", ${ }^{3,5}$ Jen-Pi Tsai ${ }^{1,3,4}$ and Cheng-Chuan Su${ }^{3,6,7^{*}}$
}

\begin{abstract}
Background: Many patients with tuberculosis (TB) are seropositive for human herpesvirus type 8 (HHV-8), and many patients with primary effusion lymphoma have high levels of HHV-8 DNA in their effusions. However, the status of HHV-8 in the effusions of patients with TB remains unclear.

Methods: Blood samples were collected from 129 patients with pulmonary TB and 129 age- and sex-matched healthy controls. Forty of the TB patients had pleural or peritoneal effusions, and 38 of these effusions were available. Both blood and effusion samples were analyzed for lymphocyte and monocyte counts and/or HHV-8 antibodies and DNA.
\end{abstract}

Results: TB patients with or without effusions had significantly greater HHV-8 seropositivity $(p=0.009)$ and titers of HHV-8 antibodies $(p=0.005)$ than healthy controls. The seropositivity and blood titers of HHV-8 antibodies were similar in TB patients with and without effusions. Among TB patients with effusions, similar percentages had seropositive plasma and seropositive effusions. Plasma samples of 6 TB patients, but none of the healthy controls, were positive for HHV-8 DNA ( $p=0.03$ ). TB patients with or without effusions had lower blood lymphocyte counts and higher blood monocyte counts than healthy controls ( $p<0.0001$ for both). TB patients with effusions had significantly lower blood lymphocyte counts than those without effusions $(p=0.035)$.

Conclusions: HHV-8 had similar seroprevalence in TB patients with and without effusions. However, TB patients with effusions had lower blood lymphocyte counts than those without effusions.

Keywords: HHV-8, Imunofluorescence assay, Kaposi's sarcoma, Primary effusion lymphoma, Tuberculosis

\section{Background}

Previous studies have consistently identified human herpesvirus type 8 (HHV-8) DNA in patients with all types of Kaposi's sarcoma (KS), and this virus is considered the etiological agent for KS $[1,2]$. Healthy individuals with HHV-8 infections typically have no symptoms or signs of disease. However, HHV-8-infected patients with diverse immunologic abnormalities [3, 4], such as those with the human immunodeficiency virus (HIV) infection, can develop KS.

HIV-infected patients have a decreased number of circulating CD4+ T-lymphocytes. Depletion of Tlymphocytes in general and CD4+ cells in particular also occurs in patients with tuberculosis (TB) $[5,6]$.

\footnotetext{
* Correspondence: sucpo@yahoo.com.tw

${ }^{3}$ School of Medicine, Tzu Chi University, Hualien, Taiwan

${ }^{6}$ Department of Clinical Pathology, Buddhist Dalin Tzu Chi Hospital, 2,

Minsheng Road, Dalin Town, Chiayi County 622, Taiwan

Full list of author information is available at the end of the article
}

Moreover, patients with HIV infection or pulmonary TB have peripheral blood mononuclear cells with impaired interferon- $\gamma$ production upon in vitro antigenic stimulation, as well as aberrant in vivo T-cell activation [7]. There is also a high HHV-8 seroprevalence in HIVinfected or pulmonary TB patients without KS [8-11]. Primary effusion lymphoma (PEL) occurs mostly in HIV-infected patients, and is associated with HHV-8 infection [12]. The HHV-8 DNA levels in effusion samples from patients with PEL are significantly higher than those in blood samples [13]. However, the presence of HHV-8 antibodies and DNA in the plasma and effusions of TB patients with pleural or peritoneal effusion has not yet been determined.

Patients with active TB are more likely to have lymphopenia and monocytosis $[7,14,15]$. Thus, we evaluated the associations of HHV-8 infection with lymphopenia and monocytosis in TB patients with and without effusions. In 
particular, we assessed the positivity and titers of HHV-8 antibodies and the positivity and levels of HHV-8 DNA in blood and effusion samples of TB patients with pleural or peritoneal effusions.

\section{Methods}

\section{Subjects and sample collection}

All patients had newly diagnosed pulmonary TB and were examined before treatment. TB was initially suspected based on clinical features and abnormalities on chest radiographs, and confirmed by identifying Mycobacterium tuberculosis from sputum cultures in LöwensteinJensen medium. Patients with abdominal fullness, fever, and even dyspnea were suspected of having peritoneal effusion. These patients were given chest and/or abdominal CT exams, with aspiration of the peritoneal effusion using an echo-guide. The collected pleural and peritoneal effusions were studied for routine biochemistry including polymerase chain reaction (PCR) and mycobacterial culture for $\mathrm{TB}$ detection, and measurement of adenosine deaminase (ADA). Definite TB effusion was indicated by positive culture or PCR results. Patients without culture or PCR positivity were reassessed 2 months after the initiation of anti-TB treatment if they had a predominance of lymphocytes, high level of serum protein, and ADA greater than $40 \mathrm{mg} / \mathrm{mL}$. Because of the high prevalence and the need for legal notification, the final TB diagnosis was discussed and determined by an official committee of Centers for Disease Control of Taiwan based on clinical information and treatment outcome. Patients with additional heart, lung, or other major diseases were excluded. The study protocol was approved by the Institutional Review Board of the Buddhist Dalin Tzu Chi Hospital (B09602001 and B09703021). All individuals provided informed written consent for participation.

Plasma samples were collected following medical examinations of 129 patients with pulmonary TB and 129 age- and sex-matched healthy controls. Forty of the TB patients had pleural or peritoneal effusions, and 38 of these effusions were available. All samples were collected in sterile tubes and centrifuged immediately at $4{ }^{\circ} \mathrm{C}$ to remove cells. Aliquots of the supernatants were frozen at $-70{ }^{\circ} \mathrm{C}$ until analysis of $\mathrm{HHV}-8$ antibodies and DNA.

The lymphocyte and monocyte counts of peripheral blood from healthy controls and TB patients were analyzed using an automated hematologic analyzer (XE-2100, Sysmex, Kobe, Japan) before plasma sample collection.

The mean ages of the 91 male controls (62.5 \pm 12.6 years) and the 38 female controls (58.9 \pm 17.2 years) were similar ( $p=0.18$; $t$ test), as were the mean ages of the 91 male TB patients $(62.3 \pm 12.6$ years $)$ and the 38 female TB patients $(58.9 \pm 17.2$ years) $(p=0.22 ; t$-test $)$. The mean ages of TB patients with effusions (60.1 \pm 17.1 years; 29 men and 11 women) and without effusion
$(61.8 \pm 12.6$ years; 62 men and 27 women) were also similar $(p=0.52 ; t$-test). All patients and controls were negative for HIV antibodies.

\section{Immunofluorescence assay (IFA) for HHV-8 antibodies}

A commercially available IFA kit (Advanced Biotechnologies Inc, Columbia, MD) was used to detect HHV-8 immunoglobulin G (IgG) antibodies against the lytic antigens in plasma and effusion samples according to the manufacturer's instructions. This assay was based on HHV-8-infected body cavity lymphoma cell lines. Plasma or effusion samples at various dilutions were brought into contact with fixed infected cells and examined microscopically using a fluorescence microscope. Samples that displayed fluorescence at a dilution of 1:40 were considered positive. Maximum HHV-8 antibody dilutions were determined by end-point IFA.

\section{DNA extraction and amplification of HHV-8 DNA}

HHV-8 DNA was extracted and PCR-amplified from each patient's plasma and effusion as reported previously [11].

\section{Chemiluminescence immunoassay for HIV antibody}

Plasma samples were assayed for antibodies to HIV-1 and HIV-2 using the Vitros anti-HIV $1+2$ reagent pack, control, and calibrator (Ortho-Clinical Diagnostics, High Wycombe, England) and the Vitros ECi immunodiagnostic system (Ortho-Clinical Diagnostics, Rochester, NY) according to the manufacturer's instructions.

\section{Statistical analysis}

A $X^{2}$ test or Fisher's exact test was used to assess the significance of between-group differences in categorical variables. The significance of differences in the means of continuous variables from two groups was determined using the $t$-test. The comparison of plasma anti-HHV-8 titers in healthy controls and TB patients was analyzed using the Mann-Whitney test. A p-value less than 0.05 was considered significant. Statistical analyses were performed using SPSS version 12.0 for Windows (SPSS, Chicago, IL).

\section{Results}

Twenty-two healthy controls $(17.1 \%, 17$ males and 5 females) and 40 TB patients (31.0\%, 29 men and 11 women) were positive for HHV-8 antibodies. The HHV-8 seropositivity rate and titers of HHV-8 antibodies were significantly greater in TB patients than in healthy controls ( $p=0.009$ by $\chi^{2}$ test and $p=0.005$ by Mann-Whitney test, respectively) (Table 1 ). The mean ages were similar in healthy subjects who were seropositive and seronegative, in TB patients who were seropositive and seronegative, and in TB patients with or without effusions $(p>0.05$ for all 3 comparisons; $t$-test). The seropositivity rate was also 
Table 1 Age, lymphocyte count, monocyte count, HHV-8 seropositivity, titer of anti-HHV-8 antibodies, and presence of HHV-8 DNA in TB patients and in age- and sex-matched healthy controls

\begin{tabular}{|c|c|c|c|c|c|c|c|c|c|c|c|}
\hline & \multirow{2}{*}{$\begin{array}{l}\mathrm{Age}^{\mathrm{a}} \\
\text { (years) }\end{array}$} & \multirow{2}{*}{$\begin{array}{l}\text { Lymphocyte } \\
\text { count }^{\mathrm{a}} / \mu \mathrm{L}\end{array}$} & \multirow{2}{*}{$\begin{array}{l}\text { Monocyte } \\
\text { count }^{2} / \mu \mathrm{L}\end{array}$} & \multirow[t]{2}{*}{ IFA (+) rate ${ }^{\mathrm{b}}$} & \multicolumn{6}{|c|}{ Anti-HHV-8 with maximal dilution } & \multirow{2}{*}{$\begin{array}{l}\text { HHV- } \\
\text { DNA }\end{array}$} \\
\hline & & & & & $1: 40$ & $1: 80$ & $1: 160$ & $1: 320$ & $1: 640$ & $1: 1280$ & \\
\hline Healthy controls & $61.4 \pm 14.1$ & $1829 \pm 495$ & $315 \pm 110$ & $17.1 \%(22 / 129)$ & 15 & 6 & 0 & 0 & 1 & 0 & 0 \\
\hline$P$ value & & $<0.0001^{\mathrm{c}}$ & $<0.0001^{\mathrm{c}}$ & $0.009^{d}$ & $0.005^{\mathrm{e}}$ & & & & & & $0.03^{f}$ \\
\hline TB patients & $61.3 \pm 14.1$ & $1291 \pm 661^{9}$ & $552 \pm 291^{9}$ & $29.7 \%(40 / 129)$ & 21 & 11 & 2 & 2 & 3 & 1 & 6 \\
\hline
\end{tabular}

HHV-8 human herpesvirus type 8, IFA mmunofluorescence assay, TB tuberculosis

${ }^{a}$ Mean \pm standard deviation. ${ }^{b}$ Positive in the IFA. ${ }^{c} t$-test. ${ }^{d} x^{2}$ test. ${ }^{e}$ Mann-Whitney test. ${ }^{f}$ Fisher's exact test. ${ }^{g}$ Lymphocyte and monocyte counts of 9 TB patients were unavailable

similar in male and female healthy controls, in male and female TB patients, and in TB patients with and without effusions $\left(p>0.05\right.$ for all 3 comparisons by $\chi^{2}$ or Fisher's exact test).

Patients with effusions $(n=40)$ and without effusions $(n=89)$ had similar HHV-8 seropositivity, titer of HHV-8 antibodies, and HHV-8 DNA positivity in plasma $\left(p=0.56\right.$ by $\chi^{2}$ test; $p=0.45$ by Mann-Whitney test; and $p=0.67$ by Fisher's exact test, respectively) (Table 2 ).

The effusion samples of $2 \mathrm{~TB}$ patients with pleural effusions were unavailable, but the plasma of 1 of these patients was positive for HHV-8 DNA (472 copies/mL) (Table 2). The positive rates and titers of HHV-8 antibodies in the plasma and effusion samples of the remaining 38 patients with effusions ( 3 with ascites and 35 with pleural effusion) were similar ( $p=0.45$ by $\chi^{2}$ test; $p=0.50$ by Mann-Whitney test, respectively) (Table 3 ). The plasma and effusion samples of the 38 patients whose effusions were available were all negative for HHV-8 DNA.

The plasma samples of 3 of the 89 patients without effusions who were negative for HHV-8 antibodies were positive for HHV-8 DNA (544, 899, and 1011 copies $/ \mathrm{mL}$ ). Moreover, 2 of the patients without effusions who were positive for HHV-8 antibodies were positive for HHV-8 DNA (1415 and 3720 copies/mL). Thus, the plasma samples of $6 \mathrm{~TB}$ patients, but none of the healthy controls, were positive for HHV-8 DNA $(p=0.03)$ (Table 1$)$.

TB patients had much lower blood lymphocyte and monocyte counts than healthy controls $(p<0.0001$ for both; $t$-test) (Table 1). However, controls who were seronegative and seropositive, and patients who were seronegative and seropositive had similar blood lymphocyte counts $(1838 \pm 501 / \mu \mathrm{L}$ vs. $1783 \pm 471 / \mu \mathrm{L}$ and $1322 \pm$ $656 / \mu \mathrm{L}$ vs. $1222 \pm 673 / \mu \mathrm{L}$, respectively; $p>0.05$ for both; $t$-test) and similar blood monocyte counts $(318 \pm 109$ / $\mu \mathrm{L}$ vs. $301 \pm 115 / \mu \mathrm{L}$ and $546 \pm 251 / \mu \mathrm{L}$ vs. $565 \pm 368 /$ $\mu \mathrm{L}$, respectively; $p>0.05$ for both; $t$-test).

TB patients with effusions had significantly lower blood lymphocyte counts than those without effusions $(1112 \pm 452 / \mu \mathrm{L} \quad$ vs. $\quad 1380 \pm 729 / \mu \mathrm{L} ; \quad p=0.035 ; \quad t$-test $)$ (Table 2). Among TB patients with and without effusions, blood lymphocyte counts were similar for those who were HHV-8 seronegative and seropositive (1125 \pm $448 / \mu \mathrm{L}$ vs. $1078 \pm 484 / \mu \mathrm{L}$ and $1428 \pm 727 / \mu \mathrm{L}$ vs. $1283 \pm$ $793 / \mu \mathrm{L}$, respectively; $p>0.05$ for both; $t$-test). Blood monocyte counts in HHV-8 seronegative and seropositive TB patients with and without effusion were also similar $(534 \pm 222 / \mu \mathrm{L}$ vs. $621 \pm 428 / \mu \mathrm{L}$ and $552 \pm 268 / \mu \mathrm{L}$ vs. $542 \pm 346 / \mu \mathrm{L}$, respectively; $p>0.05$ for both; t-test).

None of the TB patients who were positive for HHV-8 antibodies or HHV-8 DNA had clinical manifestations of HHV-8 infection, such as KS, PEL, or Castleman disease.

\section{Discussion}

The major finding of the present study is that HIVnegative individuals with pulmonary $\mathrm{TB}$, both with and without effusions, had greater seropositivity for antiHHV8 antibodies than age- and sex-matched healthy controls. These results are in line with the results of our previous study of subjects with TB pneumonia [11]. The cutoff point for HHV-8 seropositivity in the present

Table 2 Age, lymphocyte count, monocyte count, HHV-8 seropostitivity, titer of anti-HHV-8 antibodies, and presence of HHV-8 DNA in TB patients with and without effusions

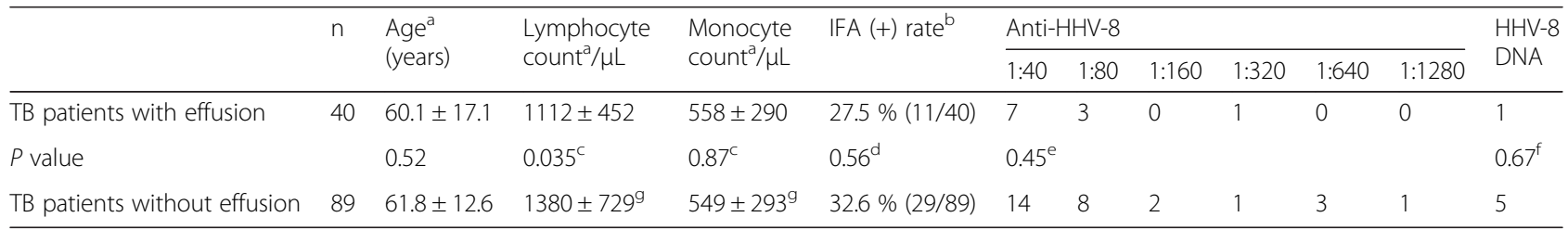

HHV-8 human herpesvirus type 8, IFA mmunofluorescence assay, TB tuberculosis

${ }^{\mathrm{a}}$ Mean \pm standard deviation. ${ }^{\mathrm{b}}$ Positive in the IFA. ${ }^{\mathrm{C}}$-test. ${ }^{\mathrm{d}} \mathrm{X}^{2}$ test. ${ }^{\mathrm{e}}$ Mann-Whitney test. ${ }^{\mathrm{f}}$ Fisher's exact test. ${ }^{\mathrm{g}}$ Lymphocyte and monocyte counts of 9 TB patients without effusions were unavailable 
Table 3 Number of TB patients who tested positive for anti-HHV-8 antibodies in plasma and in peritoneal or pleural effusion samples

\begin{tabular}{llll}
\hline & Plasma & Effusion & $P$ value \\
\hline IFA $+{ }^{\mathrm{a}}$ & $10 / 38(26.3 \%)$ & $13 / 38^{\mathrm{b}}(34.2 \%)$ & $0.45^{\mathrm{c}}$ \\
Anti-HHV-8 titers & & & $0.50^{\mathrm{d}}$ \\
$1: 40$ & 7 & 10 & \\
$1: 80$ & 3 & 3 & \\
\hline
\end{tabular}

HHV-8, human herpesvirus type 8; IFA, immunofluorescence assay;

TB, tuberculosis

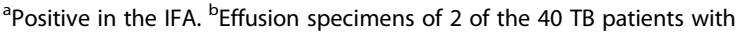
effusions were unavailable. ${ }^{c}{ }^{2}$ test. ${ }^{d}$ Mann-Whitney test

study was set at 1:40 according to the manufacturer's instructions. In our recent study of HHV-8 seroprevalence in patients with end-stage renal disease, one of the two healthy controls with IFA antibody titers of 1:160 displayed positivity in a specific enzyme-linked immunosorbent assay (ELISA) [16]. If the cutoff point for seropositivity was set at 1:160 in our previous study, then the seropositive rate among TB patients was marginally higher $(5 / 101$ vs. $0 / 101 ; p=0.059$; Fisher's exact test) [11], but in the present study the seropositive rate would remain significantly higher in patients than in controls $(8 / 129$ vs. $1 / 129 ; p=0.036$; Fisher's exact test). This discrepancy might be due to fewer subjects in the previous study.

The present study demonstrated that the seroprevalence of HHV-8 was similar among TB patients with and without effusions (27.5 vs. $32.6 \%$ ). As noted in our previous study of subjects with pulmonary TB [11], HHV-8 seroprevalence in HIV-negative TB patients with pleural effusions was lower than in HIV-negative patients with KS (52-100\%) and comparable to that in HIV-positive patients without KS (13-50\%) [8-10, 17]. This suggests that $\mathrm{HHV}-8$ infection has similar roles in TB patients with and without effusions. However, the study groups enrolled in the present and previous studies were much older than individuals who have the greatest risk for HIV infection.

Previous studies reported that the prevalence of $\mathrm{HHV}-8$ infection increases with age [18-20], and that KS is more common in elderly males [21]. Our previous comparison of TB patients and healthy controls [11] indicated similar prevalence of $\mathrm{HHV}-8$ seropositivity for males and females and similar mean ages for seropositive and seronegative subjects. In agreement, the present study demonstrated similar seropositivity for males and females, similar mean ages for seronegative and seropositive subjects, and similar seropositivity for TB patients with and without effusions.

Our previous study reported similar positivity for HHV-8 DNA in TB patients and healthy controls (4/101 vs. $0 / 101, p=0.12$ ) [11]. However, the present study indicated that HHV-8 DNA positivity was significantly greater in the TB group than in the control group $(6 / 129$ vs. $0 / 129, p=0.03)$. These discrepant findings might be attributable to smaller number of subjects in our previous study.

Our previous study of patients with cirrhosis and ascites indicated the positive rate and titers of anti-HHV-8 antibodies were much greater in plasma than ascites $(p<0.0001)$ [22]. Among cirrhosis patients, the positive rate of anti-HHV-8 antibodies in plasma samples was more than 6-fold greater than in effusion samples. By contrast, the present study indicated similar positive rates and titers of anti-HHV-8 antibodies in plasma and effusion samples among TB patients with effusions (13/38 vs. $10 / 38, p=0.45)$. The most likely reason for this difference is that TB effusions are exudates, but cirrhosis effusions are transudates [23].

HHV-8 is associated with PEL, a rare form of malignant clonal B-cell lymphoproliferation [12]. Among patients with PEL, the level of HHV-8 DNA in effusion samples is significantly higher than in blood samples [13]. In contrast, the present study found that plasma and effusion samples from the $38 \mathrm{~TB}$ patients with available effusions were all negative for HHV-8 DNA. This suggests that the role of HHV-8 is different for patients with TB and those with PEL.

The present study found that TB patients with or without effusions had lower lymphocyte counts and higher monocyte counts than healthy controls $(p<$ 0.0001 for both), in agreement with our previous study of patients with TB pneumonia [11]. Stimulated pleural macrophages can induce production of interleukin-8 (IL-8) protein, and IL- 8 in turn can induce lymphocyte chemotaxis into the pleural space [24]. Hence, lymphocyte counts are elevated in the effusions of patients with TB [25]. This may explain the finding of the present study that TB patients with peritoneal or pleural effusions had significantly lower blood lymphocyte counts than those without effusions $(p=0.035)$.

These are four limitations in the present study. First, although this is the first study to comprehensively investigate the prevalence of $\mathrm{HHV}-8$ in TB patients with peritoneal or pleural effusions, all participants were recruited from a single teaching hospital. Thus, the results may not be representative of the overall situation in Taiwan or elsewhere in the world. Second, the PCR method used in this study can only detect 5-10 copies of HHV-8 DNA, and therefore may be insufficiently sensitive for definitively establishing the presence or absence of HHV-8 DNA. The question of whether patients such as those who participated in this study have HHV-8 DNA at levels less than $5-10$ copies/mL be resolved if the detection sensitivity can be increased. Third, we did not investigate the potential for a common underlying cause of both TB and HHV8 infection, such as socioeconomic status, nutritional conditions, and health-related behaviors. Fourth, we did not 
have records of any co-morbidities or adjunct therapies in our TB population, and we expect these to be relatively common in older individuals, such as those in our study population.

\section{Conclusion}

The seropositivity and titers of anti-HHV-8 antibodies and the seropositivity of HHV-8 DNA were significantly higher in TB patients with or without peritoneal or pleural effusions than in healthy controls. The prevalence of HHV-8 in TB patients with or without effusions is comparable to that previously reported for HIVinfected patients without KS. Thus, our study suggests that TB patients with or without effusions are vulnerable to HHV-8 infection, similar to HIV-infected patients. We also found that the seropositivity and titers of antiHHV-8 antibodies and the seropositivity of HHV-8 DNA were similar for TB patients with and without effusions, suggesting a similar role for HHV-8 in these two groups of TB patients. TB patients with effusions had significantly lower blood lymphocyte counts than those without effusions. This may be attributable to IL-8 production mediated by stimulated peritoneal or pleural macrophages and the subsequent induction of lymphocyte chemotaxis into the body cavities.

\author{
Abbreviations \\ ELISA: Enzyme-linked immunosorbent assay; HHV-8: Human herpesvirus type \\ 8; HIV: Human immunodeficiency virus; IFA: Immunofluorescence assay; IL- \\ 8: Interleukin-8; KS: Kaposi's sarcoma; ORF: Open reading frame; \\ PCR: Polymerase chain reaction; PEL: Primary effusion lymphoma; \\ TB: Tuberculosis.
}

\section{Competing interests}

The authors declare that they have no competing interests.

\section{Authors' contributions}

CCS contributed to the funding and the conception, design, and conduct of the research; SMT, CLL, and MNL collected data; SMT and CCS analyzed and interpreted data and contributed to the writing of the paper; CCS and JPT performed statistical analyses; all authors participated in the critical review and interpretation of the data. All authors read and approved the final manuscript.

\section{Acknowledgements}

This study was supported by grants DTCRD96(2)-02 and DTCRD101(2)-E-07 from the Buddhist Dalin Tzu Chi Hospital, Chiayi County, Taiwan. The funder played no role in study design, in the collection, analysis, and interpretation of data; in the writing of the manuscript; and in the decision to submit the manuscript for publication.

\footnotetext{
Author details

${ }^{1}$ Institute of Medicine, Chung Shan Medical University, Taichung, Taiwan. ${ }^{2}$ Sections of Infectious Diseases and Chest Medicine, Department of Internal Medicine, Chung Shan Medical University Hospital, Taichung, Taiwan. ${ }^{3}$ School of Medicine, Tzu Chi University, Hualien, Taiwan. ${ }^{4}$ Department of Internal Medicine, Buddhist Dalin Tzu Chi Hospital, Chiayi County, Taiwan. ${ }^{5}$ Department of Family Medicine, Buddhist Dalin Tzu Chi Hospital, Chiayi County, Taiwan. ${ }^{6}$ Department of Clinical Pathology, Buddhist Dalin Tzu Chi Hospital, 2, Minsheng Road, Dalin Town, Chiayi County 622, Taiwan. ${ }^{7}$ Department of Anatomic Pathology, Buddhist Dalin Tzu Chi Hospital, Chiayi County, Taiwan.
}

Received: 4 July 2015 Accepted: 6 October 2015

Published online: 30 October 2015

\section{References}

1. Buonaguro FM, Tornesello ML, Beth-Giraldo E, Hatzakis A, Mueller N, Downing R, et al. Herpesvirus-like DNA sequences detected in endemic, classic, iatrogenic and epidemic Kaposi's sarcoma (KS) biopsies. Int J Cancer. 1996;65:25-8.

2. Su CC, Li CF, Liao YL, Lin CN L, Lu JJ. Immunohistochemical and molecular assessment of human herpesvirus type 8 in gastrointestinal tumors. J Clin Pathol. 2005:58:856-9.

3. Rady PL, Hodak E, Yen A, Memar O, Trattner A, Feinmesser M, et al. Detection of human herpesvirus-8 DNA in Kaposi's sarcomas from iatrogenically immunosuppressed patients. J Am Acad Dermatol. 1998;38:429-37.

4. Al-Khader AA, Shaheen FA. Posttransplant complications encountered in renal transplantation in the Middle East. Transplant P. 2004;36:180-3.

5. Turett GS, Telzak EE. Normalization of CD4+ T-lymphocyte depletion in patients without HIV infection treated for tuberculosis. Chest. 1994;105:1335-7.

6. Kony SJ, Hane AA, Larouze B, Samb A, Cissoko S, Sow PS, et al. Tuberculosisassociated severe CD4+ T-lymphocytopenia in HIV-seronegative patients from Dakar. SIDAK Research Group J Infection. 2000;41:167-71.

7. Hertoghe T, Wajja A, Ntambi L, Okwera A, Aziz MA, Hirsch C, et al. T-cell activation, apoptosis and cytokine dysregulation in the (Co)pathogenesis of HIV and pulmonary tuberculosis (TB). Clin Exp Immunol. 2000;122:350-7.

8. Preiser W, Szep NI, Lang D, Doerr HW, Rabenau HF. Kaposi's sarcoma-associated herpesvirus seroprevalence in selected German patients: evaluation by different test systems. Med Microbiol Immunol. 2001;190:121-7.

9. Wang YF, Lee SB, Cheng LC, Tai MH, Su IJ. Detection of serum antibodies to three different recombinant antigens of human herpesvirus 8 by immunoblotting: seroprevalence studies in Taiwan. Clin Chim Acta. 2002;320:37-42.

10. Chatlynne LG, Ablashi DV. Seroepidemiology of Kaposi's sarcoma-associated herpesvirus (KSHV). Semin Cancer Biol. 1999;9:175-85.

11. Su CC, Lai CL, Tsao SM, Lin MN, Hsieh TC, Lu JJ, et al. High prevalence of human herpesvirus type 8 infection in patients with pulmonary tuberculosis in Taiwan. Clin Microbiol Infect. 2015;21:266.e5-7.

12. Jenner RG, Maillard K, Cattini N, Weiss RA, Boshoff C, Wooster R, et al. Kaposi's sarcoma-associated herpesvirus-infected primary effusion lymphoma has a plasma cell gene expression profile. Proc Natl Acad Sci U S A. 2003;100:10399-404.

13. Marcelin AG, Motol J, Guihot A, Caumes E, Viard JP, Dussaix E, et al. Relationship between the quantity of Kaposi sarcoma-associated herpesvirus (KSHV) in peripheral blood and effusion fluid samples and KSHV-associated disease. J Infect Dis. 2007;196:1163-6.

14. Onwubalili JK, Scott GM. Immune status in tuberculosis and response to treatment. Tubercle. 1988;69:81-94.

15. Liam CK, Pang YK, Poosparajah S. Pulmonary tuberculosis presenting as community-acquired pneumonia. Respirology. 2006;11:786-92.

16. Su CC, Tsai JP, Lin MN, Hsieh TC, Tseng RC, Chu TY. High seroprevalence of human herpesvirus type 8 in patients with end-stage renal disease in Taiwan. J Clin Virol. 2013;58:89-93.

17. Miller G, Rigsby MO, Heston L, Grogan E, Sun R, Metroka C, et al. Antibodies to butyrate-inducible antigens of Kaposi's sarcoma-associated herpesvirus in patients with HIV-1 infection. N Engl J Med. 1996;334:1292-7.

18. Serraino D, Toma L, Andreoni M, Butto S, Tchangmena O, Sarmati L, et al. A seroprevalence study of human herpesvirus type 8 (HHV8) in eastern and Central Africa and in the Mediterranean area. Eur J Epidemiol. 2001;17:871-6.

19. Hudnall SD, Chen T, Rady P, Tyring S, Allison P. Human herpesvirus 8 seroprevalence and viral load in healthy adult blood donors. Transfusion. 2003:43:85-90.

20. Cunha AM, Caterino-de-Araujo A, Costa SC, Santos-Fortuna E, Boa-Sorte NC, Goncalves MS, et al. Increasing seroprevalence of human herpesvirus 8 (HHV-8) with age confirms HHV-8 endemicity in Amazon Amerindians from Brazil. J Gen Virol. 2005;86:2433-7.

21. Su CC, Lu JJ, Perng CL, Yu FT, Chiu CH. Evolution of human herpesvirus type 8-associated gastric Kaposi's sarcoma following corticosteroid treatment for asthma. J Formos Med Assoc. 2006;105:155-9. 
22. Chou AL, Huang WW, Lin MN, Su CC. Human herpesvirus type 8 in patients with cirrhosis independent of thrombocytopenia. J Clin Pathol. 2010;63:254-8.

23. Light RW. Management of pleural effusions. J Formos Med Assoc. 2000;99:523-31.

24. Pace E, Gjomarkaj M, Melis M, Profita M, Spatafora M, Vignola AM, et al. Interleukin-8 induces lymphocyte chemotaxis into the pleural space. Role of pleural macrophages. Am J Resp Crit Care. 1999:159:1592-9.

25. De Oliveira HG, Rossatto ER, Prolla JC. Pleural fluid adenosine deaminase and lymphocyte proportion: clinical usefulness in the diagnosis of tuberculosis. Cytopathology. 1994;5:27-32.

\section{Submit your next manuscript to BioMed Central} and take full advantage of:

- Convenient online submission

- Thorough peer review

- No space constraints or color figure charges

- Immediate publication on acceptance

- Inclusion in PubMed, CAS, Scopus and Google Scholar

- Research which is freely available for redistribution 